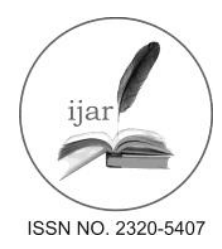

Journal homepage: http://www.journalijar.com
Journal DOI: 10.21474/IJAR01

INTERNATIONAL JOURNAL

OF ADVANCED RESEARCH

RESEARCH ARTICLE

\title{
Survey on Adaptive Front Light System.
}

Vaishali D.Todkar ${ }^{1}$ and Mrs.M.R.Bachute ${ }^{2}$.

1. Student, G.H Raisoni Instituteofengineering and TechnologyWagholi-Pune, India.

2. Faculty, G.H Raisoni Institute of engineering and TechnologyWagholi-Pune, India.

\section{Manuscript Info}

Manuscript History:

Received: 18 February 2016

Final Accepted: 19 March 2016

Published Online: April 2016

Key words:

Adaptive Front Light System (AFS),

Conventional Light System, Angle of

CurvedRoad,Sensing distance.

*Corresponding Author

Vaishali D.Todkar.

\begin{abstract}
This paper, presents an Adaptive Front Light System for better illumination of Light during curved road. Major road accidents are occurs during night time. About $25 \%$ of driving done at night time. About $55 \%$ of accidents are occurs during this periode.This is because conventional headlamp not gives the sufficient illumination of light at night time. When vehicle is driven on the curved road then, In AFS headlamps do the horizontal movement by using image processing. According to the distance between subject vehicle and incoming vehicle, AFS headlampsdoes the vertical movement.AFS improve the accuracy and reliability of the headlamp which result in human safety.

The purpose of this paper is to present survey of all the vehicles front light system and introduce latest vision based key technology. By studying disadvantage of traditional headlight system possible improvement proposal and algorithm are provided.
\end{abstract}

Copy Right, IJAR, 2016. All rights reserved.

\section{Introduction:-}

In recent decades road accidents are rapidlyincreasing. Road accidents are human tragedy. About $70 \%$ accidents are occurs in night time and $30 \%$ accidents are occurs in day time. Road safety is an issue of national concern. Accidents have negative impacts on economy, public health and public safety.

The conventional headlamp provides illumination of light only in tangent direction of the headlamp. It is not consider the turning of the road and distance between incoming vehicle and subject vehicle. Therefore driver get the incomplete and unreliable view of the road. Because of this it is necessary to do research on new technology.

Different researchers across the globe studied Adaptive Front Light (AFS) system as an innovative technology.AFS controls the light distribution according to the slope of the road and distance between incoming vehicle and subject vehicle.

The first electric headlamp was introduced in 1898 on the Columbia asia car. The concept of moving of the headlamp is actually old one. The old innovation in lighting was vertically tilt the beams introduced in 1917.Horizontal swiveling is also important in automotive industry.AFS is still inaccessible feature for the most of the car on roads today.

New developed AFS is based on image processing. This new kind of AFS system uses image recognition technology to collect the corner information from a certain distance and then it adjust the horizontal movement of the headlamp. For sensing the obstacle coming from front side ultrasonic distance sensor is used accordingly vertical movement of headlamp is takes place. 


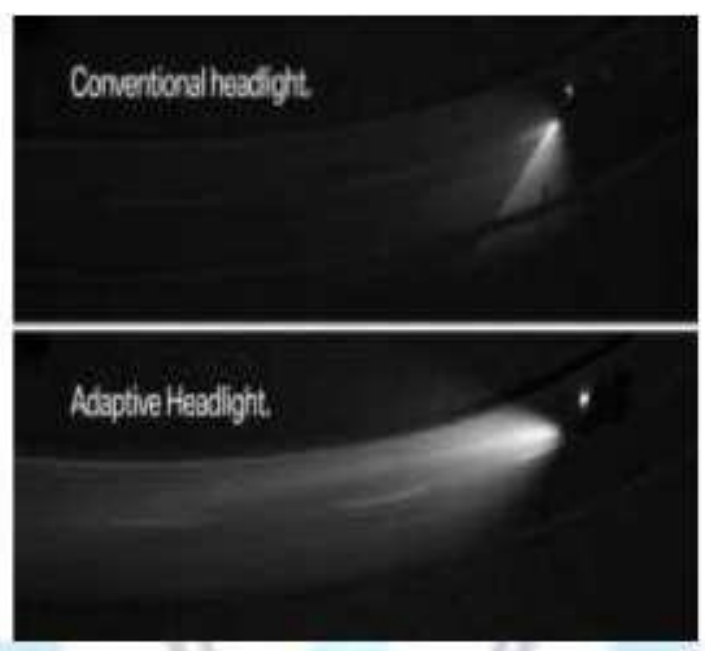

Fig 1.Conventional vs. Adaptive Headlight

Above fig1.shows the conventional vs. adaptive headlight system in which we can observe that how better illumination of light get in adaptive headlight system..

The objective to build AFS is modifying the conventional headlamp system considering cost and reliability. It will also increase the comfort and safety of the driver. Another objective is to disassemble the conventional headlight and modify projector light for beam rotation. In order to reduce cost and complexity simple framework is used to develop AFS.

\section{Motivation:-}

One of the problems exhibited by existing headlight lies in the fact that the headlight operates only one of the two modes either as high beam or low beam. Because of that in case of curved road or incoming vehicle driver get the unreliable and insufficient view of the road andprobability of the accident is increases.Modern headlight system offer the chance to improve the vision of car driver in front of their car in situation which not well served by conventional headlamp system.Espetially curved roads, intersections and the far zone of straight roads, highways at higher speed need to be illuminated better by the automatic headlamp. This can be achieve by well designed and tested adaptive front light.

\section{Literature survey:-}

MeftahHrairi and Anwar, AbuBakar[1] presented the "Development of an Adaptive Headlamp system": In this paper author described the steerable headlight by using components which are easily available in market which indirectly increase the reliability decrease product cost. They have used real time sensor to sense steering angle and vehicle speed. This paper introduces the AFS system that will modify the conventional headlamp which improves the reliability and safety. Potentiometer sensor is used to sense the steering angle of car. Pic microcontroller is used.Servo motor is used to move the headlight horizontally.

This paper described only horizontal moving of the headlamp. Therefore system only achieve the $50 \%$ of actual adaptive headlamp working.If we actually develop this system then when the headlight at right most position or leftmost position we see the obstacle clearly than conventional headlamp this is the advantage of system another advantages are system has high adaptability, lesscost, simple and dependable assembly design.

After studying the development of an adaptive headlamp system [1] author have described only horizontal moving of headlamp they have not described the vertical moving of headlamp.

YaliGuo,QinmuWu,Honglei Wang[2] presentedthe "Design And Implementation Of Intelligent Headlamp Control System Based On Can Bus": In this paper author described motion model of headlights in which turn angle of headlamps in horizontal and vertical direction can be calculated by mathematical equations. System contains hardware as well as software. System achieves the dynamic adjustment for headlamp horizontally and vertically by receiving data from CAN bus. 
The system uses the potentiometer to sense the vehicles steering angle and uses no of keys to simulate speed of car. Angle of the potentiometer sensor ranges from 0 degree to 180 degrees. It is observed that the time required for data processing is less than 10 millisecond so time delay between the potentiometer and headlight can be neglected.

After studying this paper[2] I have conclude that system is feasible and CAN bus is used as a data transmission channel. Advantages of system is fast response, stability but disadvantage is small error is present in the system because of mathematical calculations.

M.Canry,S.Cherfan,P.Lecocg,J.M.Kelada,A.Kemeny[3] presented the "Application of Real Time Lighting Simulation for Intelligent Front Lighting Studies "Inthis paper author introduce the adaptive headlight strategies which are used in Renault Automobile.They have use the real time lighting simulation.The driving simulator include driver cockpit, an image generator which provide realistic restitution of headlight distribution, a sound generator, realistic traffic and mam machine interface.This system can easily handled during switching in real time driving conditions. The system is called as Valeo Lighting system Branch which is developed by Ranault Lighting Department which includes dynamically directing additional headlamp according to the behavior vehicle,environment and road conditions.

They have used the Halogen bulbs which provides $30 \%$ more light than other previousgeneration bulbs.Because of the clear lens and complex shape uses $80 \%$ light that are previously wested on shields and bezels. They have used Xenon lightning which increasing the performance in the range: double flux and beam width, $40 \%$ reduction in use of electricity,day light colour rendering,30\% increased range.Ranault Xenonheadlamp has been installed in the safranein early 1997.

After studying this paper[3] We found that Author divide the light distribution in Motoway light,Adverse weather light,Town Light,Bending Light.Several simulation have been developed by Ranault Research Department since 1989.Lighting simulator save cost and time that are required for the headlight turning process.

ShirsathShashikant,Mechkul M.A.[4]presented the "Adaptive front Light System "In this paper author described the horizontal swings of headlamp by sensing steering angle and vertical swings of headlamp by sensing distance of incoming vehicle.Author mainly considerthe Accuracy,Consistency,Availability of components.

The aim of this system is build cost effective Adaptive Front Light System that will achieve increase safety comfort and enhance drivers visibility.The system consist of input sensors, a FPGA as head of system and motor for rotating headlights.Author suggested to use the ultrasonic distance sensor and potentiometer as a steering angle sensor.Horizontal movement achieved through potentiometer sensor and Vertical movement is achieve through ultrasonic distance sensor. By taking reference from this paper I have used the ultrasonic distance sensor for vertical movement but for horizontal I have used the image processing which reduces time required for horizontal movement of headlamp this is the advantage of AFSsystem over this system.

WeibinWu,TianshengHong,JosephMwapechileshe,ChuwenChen,JinxingGuo and Zhixiong presented[5] "Reasearch of Dynamic Steering Headlamps System Based On LIN Bus"In this paper author introduce the dynamic steering headlamp system using Lin communication module. In this system Master mode angle collection module and steering gear control module is present. Masteris obtaining the angle signal using angle sensor KMA200 and initiate communication and slave node is adjusting the front light.Master loads information in data field and send it to slave.Massage is filtered and the message of which the ID field and local ID is consistent,data field is read and PWM is used to control the steering gear parameter.

System has a more Accuracy and improves safety. Technology can be used for luxury cars not used in mid and low car because of his cost. They have observed that $13.7 \%$ of traffic jams at night in china is due to poor visibility.

Recently no of car manufacturer and semiconductor companies have jointly developed low speed network communication standard LIN to make the car more comfortable and more convenient.CAN system can be replace LIN system with reduce system cost and improve system performance. Aim of this system is to design cheap and compatible LIN which can link the car with AFS system. 


\section{Need:-}

The most important and safety functions of vehicle are headlamp. There is large disparity between daytime and night time traffic fatalities. So the light distribution of headlamps should be able to fulfill all needs such as, no glare, sufficient illumination of light to avoid accidents.

In our daily life, while using vehicle at night we face a problem of blind spot when turning at corner. Headlamps are parallel to body of vehicle so while turning to left as well as right light illumination is tangential and insufficient for the driver so there is need of adaptive front light system in which when vehicle turns left or right at corners of the road headlight also moves according to turn and will get better light illumination. By using this adaptive front light system we can avoid accidents up to the $50 \%$.

\section{Proposed work:-}

After studying literature survey and understanding need globallythese papers are modified and a novel technique is build called adaptive front light system

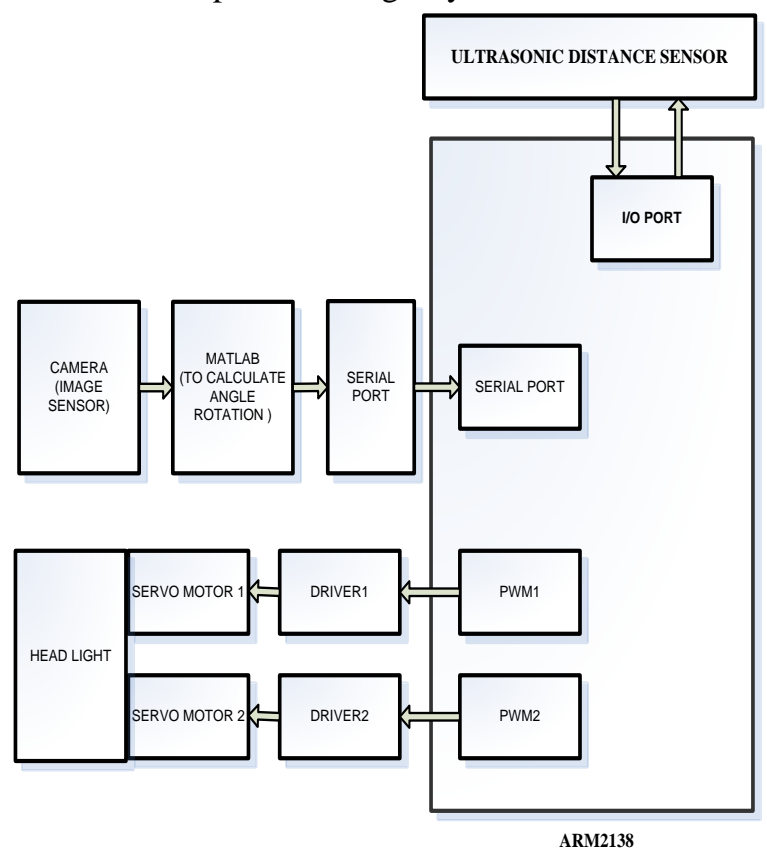

Fig 2: Block diagram of Adaptive Front Light System

The block diagram of proposed system isshown in figure2. In this system camera is used to detect information about corner.Matlab is used to build mathematical model of this system. Image processing hardware is used as sensor for detecting radius of curvature of road.

\section{Horizontal movement of headlamp:-}

Matlab is used to configure the camera for manual trigger. When the image will be captured matlab code will trigger the camera and obtain live image from camera.This image will go process of operations like preprocessing, colors conversion,segmentation,feature extraction and decision this result of road curvature will be send to microcontroller by serial port. After receiving matlab result microcontroller will calibrate the angle and will change the PWM signal fed to servomotor. Camera and horizontal servo motor form sensor actuator loop. Here Hough transform for image processing is used. FromHough transform rotation angle for the turn will be calculated. Ifroadis turning right then $\mathrm{R}$ is send to the robot and if road is turning left then $\mathrm{L}$ is send to the robot. In this way matlab processing is used in this system for image processing to find angle of road so that vehicle can done adjustment in advance to avoid obstacles

\section{Vertical movement of the headlamp:-}

Ultrasonic distance sensor is used for vertical movement of headlamp. This sensor is work on principle similar to radar and sonar just like measuring instrument that evaluate attribute of a goal by decoding the echoes from radio 
waves and sound waves severally. It generates high frequency sound waves and evaluates the echo that is received by the device. Sensor calculates fundamental measures between causing the signal and receiving the echo to determine the space to an object. When incoming vehicle is comes in range of ultrasonic sensor it transfers sound waves towards the vehicle. Then upon receiving the eco turn the sound waves in to electric energy which can be measured and transfer to the microcontroller. Then this electric voltage value is given to the PWM of the vertical servo and there is vertical movement of the headlamp is takes place.

\section{Components used:-}

LPC 2138 an ARM7 series microcontroller is used to control the motor. Motor is used to rotate the headlight mounted above it.There two types of motor available one is a stepper motor and another one is a servomotor by using these motor we can swing the headlamp but they differ in no of ways. Servomotor requires the analog feedback to control the system. In making choice between stepper motor and servomotor no of issues are considered. It is mainly depend on application which we have to run. Servomotor is more suitable for the AFS design because servomotor have built in feedback circuit.

PWM is nothing but the pulse width modulation.PWM is a technique to controlling analog circuits with processors digital output.PWM is used in wide variety of applications ranging from communication, measurement to power control and conversion.

LPC 2138 ARM7 series microcontroller is used to control the motor.LPC 2138 used 16/32 Bit and has 512KB of internal flash and 32+8K RAM. It has inbuilt 14 channel and 10 bit ADC,6 channel and 10 bit PWM and high speed of $60 \mathrm{MHz}$.Choice of controller is determined by easy availability, affordable price and reliability.

\section{Modification in the circuit:-}

As I have referred all the related papers of adaptive front light system but by using image processing in modified adaptive front light system, I tried to make the AFS more reliable and high responsive. Camera is used as a sensor for the horizontal movement of the headlamp. It can be modified and used as movable surveillancecamera. It can be used for the robotic vision and robotic ARM movement.

Sensor used for the vertical movement of the headlamps isultrasonic distance sensor which is easily available and affordable. By vertical movement of headlamp driver get the better illumination of light and driver can see the obstacle more clearly.

This AFS system definitely helps to improve road illumination. Because of using image processing will get more range vision in curve compared to traditional AFS.Arrival of this new technology will give opportunities for new suppliers to enter in a market.

\section{Conclusion:-}

As discussed above at present existing AFS is not sufficient for driving at night because of disadvantages like lag and precision to avoid this disadvantage this paper put forward an advanced AFS using camera as sensor traditional AFS has greater error in getting curvature radius this new AFS system provides curvature information superior than traditional.

Camera is used to detect real time information of curved road. In this movement of light beam is earlier than turning steering wheel by driver. The AFS system based on image processing proposed. This AFS system has high sensitivity and low cost.

The future work mainly concentrate to invent a comprehensive AFS system which can be suitable for complex road condition including related to this paper. Different conditions can be road surface water, highway, rural road, urban road and so on.

\section{Acknowledgment:-}

Author is extremelythankfulto researchguide and P.G.Co-ordinator"Mrs.M.R.Bachute", G.H.Raisoniinstituteofengineering andtechnology, Puneforconsistentguidance, inspirationand hervaluablesupport. Iam alsograteful toourcollege principal- "Prof.Dr.R.D.kharadkar"and HOD of electronics\& telecommunication department"Prof.N.B.Hulle" fortheirvaluable supportandguidance 


\section{References:-}

1. MeftahHrairi and Anwar B. Abu Bakar"Development of an Adaptive Headlamp Systems"International Conference on Computer and Communication Engineering” (ICCCE 2010), 11-13 May 2010, Kuala Lumpur, Malaysia,IEEE

2. YaliGuo, Qinmu Wu, HongleiWang "Design and implementation of intelligent Headlampcontrol system based CAN Bus"978-1-4673-0199-2/12/\$31.00 @2012 IEEE

3. M.Canry,S.Cherfan,P.Lecocg,J.M.Kelada,A.Kemeny“Application of Real Time Lighting Simulation for Intelligent Front Lighting Studies” 93012 BobignyCedex, - France

4. ShirsathShashikant,MechkulM.A.“Adaptive front Light System”IOSR Journal of Electronics and Communication Engineering (IOSR-JECE).

5. WeibinWu,TianshengHong,JosephMwapechileshe,ChuwenChen,JinxingGuo and Zhixiong "Reasearch of Dynamic Steering Headlamps System Based On LIN Bus” Journal of computers, vol. 6, no. 12, december 2011

6. Weifeng Wang, Qing Wu, Zhiyong Lu, Xiumin Chu, “Control Model and Simulation for Adaptive Front light System of Vehicle on Curve Roads"2010.,IEEE

7. T. Aoki, H. Kitamura, K. Miyagawa, and M. Kaneda, (1997).“Development of active headlight system, ”(SAE Technical Paper Series No. 970650).Warrendale, PA: Society of Automotive Engineers, 1997.,IEEE

8. H. Hogrefe and R. Neumann, "Adaptive Light Pattern - A new way to improve Light Quality,"SAE 970644, 1997,IEEE

9. Dr Joachim Damasky, Dr ArnHosemann, "The Influence of the Light Distribution of Headlamps on Drivers Fixation Behaviour at Night", SAE 980319, 1998.,IEEE

10. C.K.Chan,K.W.E.Cheng,S.L.Ho,"Development of packaging and Electrical Interfacing for Electrical Vehicles",Proc PESA '06, pp.234-24-.Nov 2006.

11. Varghese,Cherian,Shankar,Umesh"Passenger Vehicle Occupant Fatalities by Day and Night - A Contrast". Traffic Safety Facts, Research Note (DOT HS 810 637) Retrieved 2010-12-30,May2007.

12. "EUREKA AFS task force". Memagazine.org. Retrieved 2012-01-31 July 03, 2012 Status Report of HLDI. HLDI is a nonprofit research organization that publishes insurance loss statistics on most car, SUV, pickup truck and motorcycle models on U.S. road. 